\title{
Mechanism Investigation of Hyaluronidase-Combined Multistage Nanoparticles for Solid Tumor Penetration and Antitumor Effect
}

This article was published in the following Dove Press journal: International Journal of Nanomedicine

\author{
Enrui Chen ${ }^{1}, *$ \\ Shangcong $\mathrm{Han}^{1}$ * \\ Bo Song ${ }^{2}$ \\ Lisa $\mathrm{Xu}^{\prime}$ \\ Haicheng Yuan ${ }^{2}$ \\ Mingtao Liang ${ }^{3}$ \\ Yong Sun (1)
}

'Department of Pharmaceutics, School of Pharmacy, Qingdao University, Qingdao, People's Republic of China; ${ }^{2}$ Department of Neurology, Qingdao Central Hospital, Qingdao, People's Republic of China; ${ }^{3}$ Department of Pharmaceutics, School of Biomedical Science and Pharmacy, University of Newcastle, Newcastle, NSW, Australia

*These authors contributed equally to this work
Correspondence: Yong Sun

Department of Pharmaceutics, School of Pharmacy, Qingdao University, Qingdao 26602I, People's Republic of China

Tel +86-532-82991203

Email sunyong@qdu.edu.cn
Background: Hyaluronic acid (HA) is a major component of extracellular matrix (ECM) and its over expression in tumor tissues contributes to the increase of interstitial fluid pressure (IFP) and hinders the penetration of nanoparticles into solid tumors.

Materials and Methods: We here reported a tumoral microenvironment responsive multistage drug delivery system (NPs-EPI/HAase) which was formed layer by layer via electrostatic interaction with epirubicin (EPI)-loaded PEG-b-poly(2-(diisopropylamino)ethyl methacrylate)-b-poly(2-guanidinoethylmethacrylate) (mPEG-PDPA-PG, PEDG) micelles (NPs-EPI) and hyaluronidase (HAase). In this paper, we focused on the hyaluronidasecombined nanoparticles (NPs-EPI/HAase) for tumor penetration in tumor spheroid and solid tumor models in vitro and in vivo.

Results: Our results showed that NPs-EPI/HAase effectively degrade the HA in ECM and facilitate deep penetration of NPs-EPI into solid tumor. Moreover, NPs-EPI mainly employed clathrin-mediated and macropinocytosis-mediated endocytic pathways for cellular uptake and were subsequently directed to the lysosomes for further drug release triggered by proton sponge effect. Compared with NPs-EPI, the HAase coating group showed an enhanced tumoral drug delivery efficacy and inhibition of tumor growth.

Conclusion: Overall, our studies demonstrated that coating nanoparticles with HAase can provide a simple but efficient strategy for nano-drug carriers to enhance solid tumor penetration and chemotherapeutic efficacy.

Keywords: hyaluronidase, nanoparticles, tumor penetration, ECM

\section{Introduction}

Tumor heterogenicity and poor tumor penetration remain huge challenges for nanoparticles (NPs) to deliver anti-cancer drugs. ${ }^{1}$ Compared with normal tissues, solid tumors tend to display dense extracellular matrix (ECM), abnormal tumor vasculature and lymphatic system, as well as elevated tumor interstitial fluid pressure (IFP), which intrinsically hinders the transport of NPs to the deeper regions of tumors and restrict the treatment options of NPs. Moreover, the poor treatment capacity of tumor cells in the internal region of the tumor may further lead to tumor recurrence and metastasis. ${ }^{2,3}$

Recent years, researchers have reported many novel strategies to attenuate delivery barriers and enhance tumor penetration ability. Firstly, tumourmicroenvironment-responsive nanoparticles and size-shrinkable nanocarriers have made encouraging progress in overcoming the above nano-drug treatment 
barriers. ${ }^{4-8}$ Secondly, most tumor vessels were compressed and dysfunctional due to the increased IFP in tumors, which remains the major barrier for NPs delivery. Tumor vasculature normalization strategy is also an effective way to enhance tumor penetration of nanoparticles..$^{9,10}$ Thirdly, tumor ECM is composed predominantly of hyaluronic acid, collagen and fibronectin, which may result in considerable barriers to deep penetration of nanoparticles. ${ }^{11}$ By functionalizing NPs with hyaluronidase, collagenase or bromelain to degrade ECM may be a feasible method to enhance tumor permeability. ${ }^{12}$ Eikenes and coworkers confirmed that collagenase, hyaluronidase, and relaxin increased the diffusion coefficient of the 2-MDa FITCdextrans in the spheroids. ${ }^{13}$

As one of the major components of extracellular matrix, hyaluronic acid (HA) is a non-sulphated glycosaminoglycan and its accumulation contributes to the increase of interstitial fluid pressure in tumors thus reducing nanomedicine diffusion. ${ }^{14}$ The accumulation of HA into the stroma of various human tumors depends on tumor type, malignant melanomas and squamous cell carcinomas with low HA contents. ${ }^{15}$ The accumulation of HA in tumor tissue also interferes the contact between tumor cells, recruits tumor-related macrophages, promotes epithelial-mesenchymal transition and is also related to tumor resistance. ${ }^{16,17}$ In addition to providing a hydrated gel-like matrix to support the tumor growth, HA is intimately involved in the biology of cancer, modulates intracellular signaling pathways, cell proliferation, motility and invasive properties of tumor cells, and it also can create a specific microenvironment that is favorable for tumor angiogenesis, invasion, and metastasis. ${ }^{18-21}$ Hyaluronidase (HAase) is a family of enzymes that degrade HA and increase the permeability of tissue to fluids. Intratumoral injection of HAase was able to decompose the ECM and decrease IFP in a concentration-dependent manner. ${ }^{22}$ For many years, HAase has been employed as adjuvant chemotherapy to modulate the tumor microenvironment and enhance the penetration of cancer drugs. ${ }^{23}$

In our previous studies, we have reported that after coating with HAase, drug-loaded nanoparticles showed enhanced tumoral accumulation. ${ }^{24}$ In this study, we investigated the cellular uptake and intracellular drug release for EPI-loaded mPEG-PDPA-PG (PEG-b-poly(2-(diisopropylamino)ethyl methacrylate)-b-poly(2-guanidinoethylmethacrylate), PEDG) nanoparticles and the mechanism of NPs-EPI/HAase for tumor spheroid and solid tumor penetration. As shown in
Figure 1, NPs-EPI/HAase was formed by electrostatic interaction between cationic EPI-loaded nanoparticles (NPs-EPI) and the anionic HAase. By degrading the HA in ECM, HAase could improve the penetration of NPs-EPI into solid tumor thus enhancing the uptake by cancer cells. The PDPA segment of PEDG has ideal $\mathrm{pH}$-responsive capability, which provides a rapid drug release triggered by proton sponge effect in lysosome after internalization. ${ }^{25,26}$ Combining the deep penetration ability and $\mathrm{pH}$-sensitive drug release ability, NPs-EPI /HAase nanoplexes showed enhanced inhibition of tumor growth in vivo.

\section{Materials and Methods Materials}

The chain transfer agent S-1-dodecyl-S-( $\alpha, \alpha^{\prime}$-dimethyl-a"acetic acid)trithiocarbonate conjugated mPEG (mPEGRAFT, $M w=2000$ ) was provided by Professor Dong Anjie of Tianjin University; All the materials used in the cell culture study were obtained from Sigma-Aldrich (St. Louis, USA). DAPI, anti-fluorescence quenching sealing tablet, chlorpromazine hydrochloride and amiloride hydrochloride were purchased from Solarbio (Beijing, China). Biotinylated HA binding protein was obtained from EMD Millipore (CalBioChem). Alexa Fluor 488conjugated streptavidin was obtained from Biolegend (San Diego, USA). Anti-collagen I rabbit pAb, anti- $\alpha-$ SMA mAb, anti-CD31 rabbit pAb, CY3 conjugated goat anti-rabbit IgG and CY3 conjugated goat anti-mouse IgG were obtained from Servicebio (Wuhan, China). HepG2 cells were purchased from American Type Culture Collection (ATCC, Manassas, VA, USA). The male athymic nude mice (nu/nu CD-1, 6 weeks old, 18-22 g) were purchased from Beijing Vital River Laboratory Animal Technologies Co. Ltd (Beijing, China) and maintained at $25^{\circ} \mathrm{C}$ with free access to food and water. All animal experiments had been approved by the Animal Care Ethics Committee of Qingdao University (Qingdao, China) and were carried out in compliance with the Animal Management Rules of the Ministry of Health of the People's Republic of China (document no. 55, 2001).

\section{Preparation and Characterisation of Nanoparticles}

To prepare the EPI-loaded micelles (NPs-EPI), polymer mPEG-PDPA-PG ( $8 \mathrm{mg}$ ) and EPI ( $2 \mathrm{mg}$ ) were dissolved in 800 and $200 \mu \mathrm{L}$ DMSO separately and then mixed. The solution was subsequently added dropwise to $10 \mathrm{~mL}$ of 

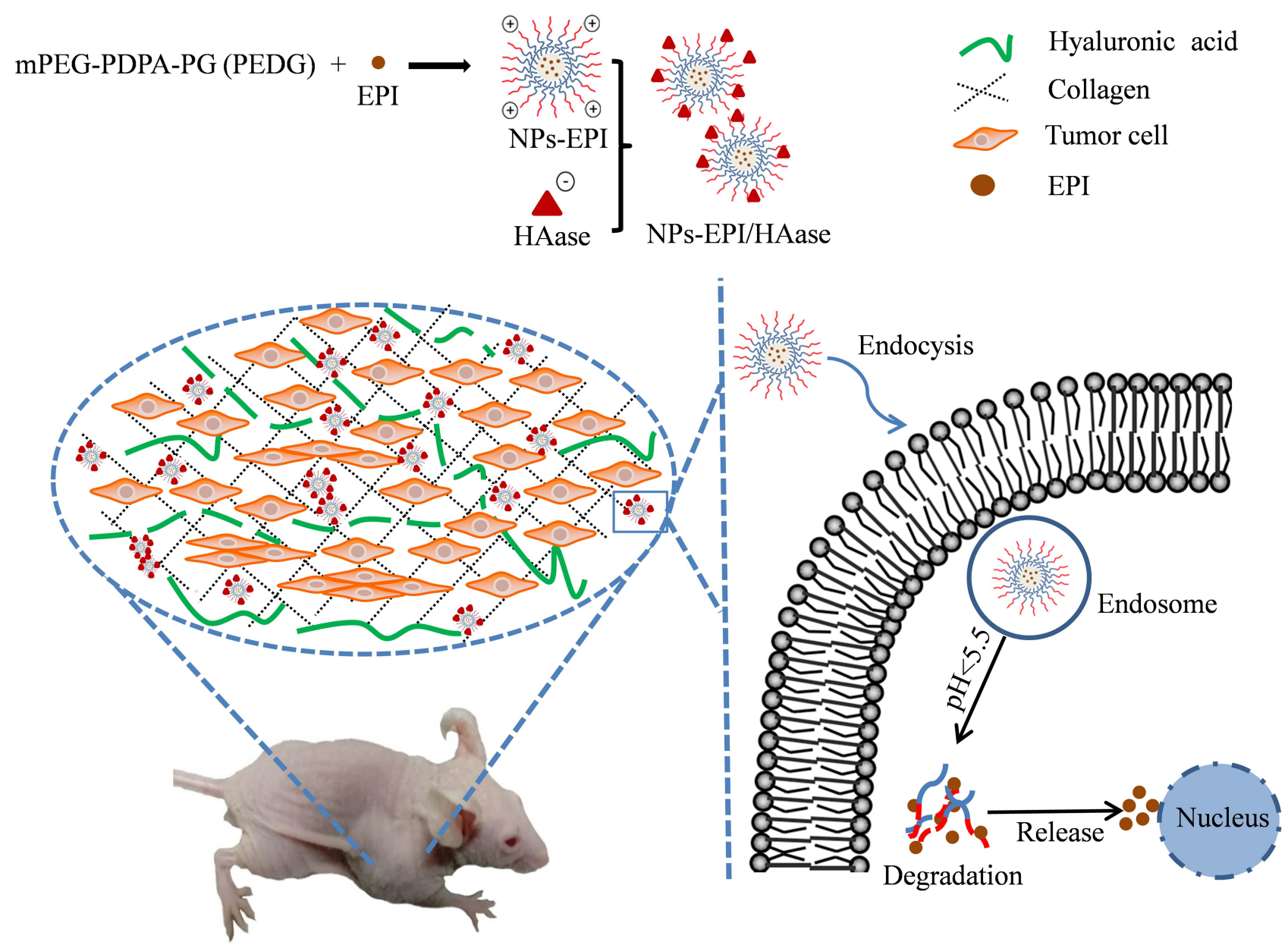

Figure I Preparation of hyaluronidase-combined nanoparticles and mechanism of penetration into solid tumor.

ultrapure water under vigorous stirring. The final mixture was stirred overnight and dialysed $(2.5 \mathrm{~L}, 24 \mathrm{~h}$; $\mathrm{Mw}=3500$ ) to remove DMSO and free drug EPI. Blank micelles were prepared by same method at a polymer concentration of $1 \mathrm{mg} / \mathrm{mL}$. Fluorescently labelled micelles (NPs-DiD) were also prepared with $\mathrm{DiD}$ at a weight ratio of $0.2 \%$.

To prepare NPs-EPI/HAase, HAase powders were dissolved in $1 \mathrm{~mL}$ of PBS and the solution was added dropwise to $1 \mathrm{~mL}$ of NPs-EPI solution $(1 \mathrm{mg} / \mathrm{mL})$ while mixing using a vortexer. The nanocomposite solution (NPs-EPI/HAase) was formed by electrostatic interaction between cationic NPs-EPI and the anionic HAase.

Particle size and zeta potential of NPs-EPI and NPsEPI/HAase were determined using Malvern Zeta Sizer Nano ZS90 (Malvern, UK). The morphology of NPs-EPI and NPs-EPI/HAase was visualized with transmission electron microscope (TEM, JEM2010, JEOL). The encapsulation efficiency (EE \%) and loading capacity (LC \%) of
EPI in NPs-EPI were quantified by measuring the fluorescence intensity of EPI (excitation at $488 \mathrm{~nm}$ and emission at $588 \mathrm{~nm}$ ), and the fluorescence intensity was read by Flex Station 3 multifunctional enzyme standard instrument workstation (MD, USA). The EE \% and LC \% were calculated according to the following equations:

$$
\begin{gathered}
\mathrm{EE} \%=\frac{\text { Amount of EPI in the polymermicelles }}{\text { Total amount of EPI added }} \times 100 \% \\
\mathrm{LC} \%=\frac{\text { Amount of EPI in polymer micelles }}{\text { Weight of polymer micelles }} \times 100 \%
\end{gathered}
$$

\section{Hemolysis Assays}

Hemolysis assay was performed to examine the potential toxicity of mPEG-PDPA-PG polymer on red blood cells (RBC); 4\% RBC suspension was first prepared with phosphate buffer solution ( $\mathrm{pH} 7.4$ ) and $800 \mu \mathrm{L}$ of $4 \% \mathrm{RBC}$ suspension was then mixed with $200 \mu \mathrm{L}$ of NPs at varied 
concentrations of $0.1,0.2,0.5$, and $1 \mathrm{mg} / \mathrm{mL}$, respectively. Triton-X-100 $(200 \mu \mathrm{L})$ was used as the positive control whereas the negative control was physiological saline. All samples were incubated $60 \mathrm{~min}$ at a temperature of $37^{\circ} \mathrm{C}$. The samples were finally centrifuged at $3000 \mathrm{rpm}$ for 10 min and the OD was measured at $545 \mathrm{~nm}$ to denote the hemolytic percentage. The hemolysis rate was calculated using the below mentioning formula:

Hemolysisrate $(\%)=$

Experiment group A value - negative control group A value

$\overline{\text { Positive control group A value }- \text { negative control group A value }} \times 100 \%$

\section{Cellular Internalization}

For initial study of cellular internalization, NPs-EPI was incubated with tumor spheroids at an EPI concentration of $8 \mu \mathrm{g} / \mathrm{mL}$ for $4 \mathrm{~h}$. The tumor spheroids were grown as our previous reported procedure. ${ }^{27}$ Briefly, HepG2 cells were seeded in 96-well plates at a density of $8 \times 10^{3}$ per well precoated with $50 \mu \mathrm{L}$ of $1 \%$ low melting point agarose. $^{28}$ Cells were cultured to obtain multicellular spheroids (400-500 $\mu \mathrm{m}$ in diameter) for subsequent studies. After incubation with NPs-EPI, tumor spheroids were rinsed three times with cold PBS and processed for image analysis by TEM.

To investigate the internalization pathways of NPs-EPI, HepG2 cells were seeded into 6 -well plates with $2 \times 10^{5}$ cells per well and incubated for 24 hours. The cells were then pre-treated with different endocytic inhibitors (chlorpromazine hydrochloride $20 \mu \mathrm{g} / \mathrm{mL}$, amiloride hydrochloride $200 \mu \mathrm{g} / \mathrm{mL}$ ) at $37^{\circ} \mathrm{C}$ for 1 hour. Subsequently, NPs-EPI (EPI $8 \mu \mathrm{g} / \mathrm{mL}$ ) were added and incubated at $37^{\circ} \mathrm{C}$ for 2 hours. Finally, the cells were washed three times with cold PBS and harvested for flow cytometry to determine the fluorescence intensity of EPI $(\mathrm{Ex}=488, \mathrm{Em}=588)$. Cells pre-treated with $\mathrm{PBS}$ at $37^{\circ} \mathrm{C}$ served as the positive control group. ${ }^{29}$ Internalization assays were also performed in the absence of endocytic inhibitors at $4{ }^{\circ} \mathrm{C}$ when indicated.

\section{Tumor Spheroid Penetration}

Tumor spheroids were treated with NPs-EPI or NPs-EPI /HAase (combined with 0.05, 0.1, 0.5, $1.0 \mathrm{mg} / \mathrm{mL}$ HAase, respectively) in EPI concentration of $8 \mu \mathrm{g} / \mathrm{mL}$ for a predetermined period $(1 \mathrm{~h}, 4 \mathrm{~h}$, and $8 \mathrm{~h})$. After incubation, tumor spheroids were rinsed with cold PBS and processed for subsequent analysis by confocal microscopy (Nikon, Japan) and fluorescence inverted microscopy (Olympus, Japan).
To investigate HA degradation in ECM, tumor spheroids were incubated with NPs or NPs/HAase (HAase $0.5 \mathrm{mg} / \mathrm{mL}$ ) for 8 hours. Tumor spheroids were subsequently gathered, fixed with $4 \%$ paraformaldehyde, frozen and sectioned at $10 \mu \mathrm{m}$ thick. Spheroid sections were then stained with HA binding protein (HABP) diluted at 1:100 (0.5 $\mu \mathrm{g} / \mathrm{mL})$ and visualized with Alexa Fluor 488conjugated streptavidin $(\mathrm{Ex}=488, \mathrm{Em}=519)$ diluted at 1:200. The cell nuclei were stained with DAPI for $10 \mathrm{~min}$.

\section{In vivo Tumor Penetration}

To develop in vivo tumor penetration model, male athymic nude mice were subcutaneously inoculated with HepG2 cells $\left(1 \times 10^{7}\right.$ cells $/ 200 \mu \mathrm{L}$ of $1: 1$ mixture of PBS and matrix glue) in the axilla of the right forelimb. When the volume of HepG2 tumors reached approximately $200 \mathrm{~mm}^{3}$, the mice were weighed and randomly divided into four groups $(n=3)$. Each group was treated by peritumoral injection of either saline (control), EPI, NPs-EPI or NPs-EPI/HAase on days 8, 11, 14, 17, and 20 after inoculation of HepG2 cells to construct tumor penetration models. The dose of EPI was $3 \mathrm{mg} / \mathrm{kg}$ and the concentration of HAase combined with NPs-EPI was $3 \mathrm{mg} / \mathrm{mL}$. The body weight and tumor size of mice were monitored during the construction of the penetration model to illustrate the penetration efficacy of different formulations. Tumor volume $(\mathrm{V})$ was calculated according to $\mathrm{V}=0.5 \times \mathrm{L} \times$ $\mathrm{W}^{2}$ (L: length; W: width).

The tumor penetration behaviour of NPs was analysed by confocal microscopy (Nikon, Japan). Due to the excitation wavelengths of EPI and Alexa Fluor 488-conjugated streptavidin overlapped, we choose $\mathrm{DiD} \quad(\mathrm{Ex}=644$, Em=665) instead of drug EPI. When HepG2 tumors reached a size of approximately $300-400 \mathrm{~mm}^{3}$, saline (control), NPs-DiD or NPs-DiD/HAase (HAase $3 \mathrm{mg} /$ $\mathrm{mL})$ were administered by peritumoral injection $(n=3)$. At $4 \mathrm{~h}$ or $12 \mathrm{~h}$ post injections, tumors were harvested and fixed with $4 \%$ paraformaldehyde, frozen and sectioned at $10 \mu \mathrm{m}$ thick. HA binding protein (HABP) diluted at 1:100 $(0.5 \mu \mathrm{g} / \mathrm{mL})$ was used for HA staining and visualized with Alexa Fluor 488-conjugated streptavidin diluted at $1: 200 .^{30}$ The spatial distribution of NPs-DiD was also analysed by confocal microscopy (Nikon, Japan).

To further investigate the penetration mechanism of NPs-EPI/HAase, the mice from each study group of tumor penetration model that we constructed was sacrificed 2 days after the last dosing (day 23 post HepG2 cell inoculation), we analysed the variation of tumor HA and 
collagen I, the density and physiologic state of tumor vasculatures and activity of cancer-associated fibroblasts (CAFs) and quantified with Image J. First, a part of the tumors was frozen and sectioned for HA staining analysis. Then, $5 \mu \mathrm{m}$ thick sections of paraffin-embedded tumor tissues were prepared for CD31, $\alpha$-SMA, and collagen I stainings and samples analysed by confocal microscopy.

\section{Histological and Immunohistochemical Analysis}

The mice were executed two days after the last dosing (day 23 post HepG2 cell inoculation). The tumors were collected, fixed in 4\% paraformaldehyde and embedded in paraffin, and $5 \mu \mathrm{m}$ thick sections were prepared. ${ }^{31}$ Subsequently, these sections were harvested for H\&E and Ki-67 antigen staining and imaged with an inverted fluorescence microscopy (Olympus, Japan). H\&E staining was employed for histological analysis to determine the tissue damage and Ki-67 antigen staining were used to estimate the cell proliferation status of tumors. ${ }^{32}$

\section{Statistical Analyses}

All data were analysed using GraphPad Prism 6.0 software and the results presented as mean $\pm \mathrm{SD}$ values. Statistical analysis was conducted using a two-tailed Student's $t$-test, in conjunction with one-way analysis of variance (ANOVA). Differences were considered statistically significant at $P<0.05$.

\section{Results and Discussion \\ Preparation and Characterization of NPs-EPI and NPs-EPI/HAase}

Well-defined mPEG-PDPA-PG (PEDG) polymer was synthesized (Figure S1, Supporting Information) and characterized (Figure S2A and B) according to our previously reported methods and used to prepare cationic micelles and complex with HAase. ${ }^{24}$ These NPs had been confirmed to have no apparent cytotoxicity by MTT assay. ${ }^{24}$ In the current study, we further examined their hemolytic toxicity since it is critical for application in intravenous injection. As shown in Figure $2 \mathrm{G}$, the hemolysis ratio of NPs was $2.2 \%$ at a concentration of $1 \mathrm{mg} / \mathrm{mL}$, which was below the safety mark of $5 \%$ indicating our samples were with low risk of hemolysis. ${ }^{33}$

After drug loading, dynamic light scattering (DLS) measurement showed that NPs-EPI and NPs-EPI/HAase had intensity averaged particle sizes of $96.6 \pm 0.4 \mathrm{~nm}$ and
$117.1 \pm 0.5 \mathrm{~nm}$, respectively (Figure $2 \mathrm{~A}$ and $\mathrm{D}$ ). The surface charge ( $\zeta$ potential) of NPs-EPI and NPs-EPI/HAase (HAase $0.5 \mathrm{mg} / \mathrm{mL}$ ) was $34.6 \pm 0.4 \mathrm{mv}$ and $19.6 \pm 0.4 \mathrm{mv}$ (Figure 2B and E), respectively. TEM images showed that both NPs-EPI and NPs-EPI/HAase had regular spherical shape and smooth surface (Figure 2C and F). The encapsulation efficiency (EE \%) and loading capacity (LC \%) of EPI in NPs-EPI were $40.57 \pm 0.62 \%$ and 8.28 $\pm 0.13 \%$, respectively.

\section{Mechanism of Cellular Internalization}

Sections of spheroids were imaged by TEM to study the cellular internalization of NPs-EPI. As shown in Figure 3A and $\mathrm{B}$, the TEM micrographs revealed that NPs-EPI were trapped in membrane-bound vesicles, suggesting the formation of early endosomes that possibly result from endocytosis. NPs-EPI were also observed in intracellular lysosome after incubation (Figure 3C). All these results indicate that NPs-EPI likely enter into cells by an endocytic pathway. The involvement of different endocytic pathways has been further investigated using different inhibitors of endocytosis: chlorpromazine $(\mathrm{CPZ})$ for clathrin-mediated endocytosis and amiloride for actin-dependent macropinocytosis. ${ }^{34,35}$ As shown in Figure 3D and E, CPZ treatment and amiloride treatment inhibited cellular uptake of NPs-EPI, indicating that the clathrin-mediated pathway and macropinocytosis played key roles in the internalization process of NPs-EPI. Furthermore, the fluorescence of cells exposed to NPs also considerably decreased when the internalization experiment was performed at $4^{\circ} \mathrm{C}$, corresponding to a reduced uptake of NPs. This indicated that the uptake of NPs in HepG2 cells was mainly an energy-dependent process, as observed in various cell types.

NPs-EPI mainly employed clathrin-mediated and macropinocytosis-mediated endocytic pathways for cellular uptake and were subsequently transferred to the lysosomes for further drug release triggered by proton sponge effect; thus, EPI could release from the NPs. It has been reported that cargos internalized by cells via the clathrinmediated pathway and macropinocytosis were mostly directed to the lysosomes, which is consistent with our observation in TEM micrograph as well as previous findings from co-localization study. ${ }^{24}$

\section{Tumor Spheroids Penetration Efficiency}

Tumor spheroids could simulate the in vivo status and functions of tumor, including the production of ECM production and other proteins, therefore, it has been widely used to 
A

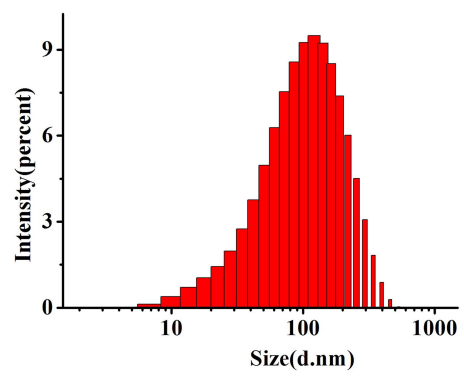

D

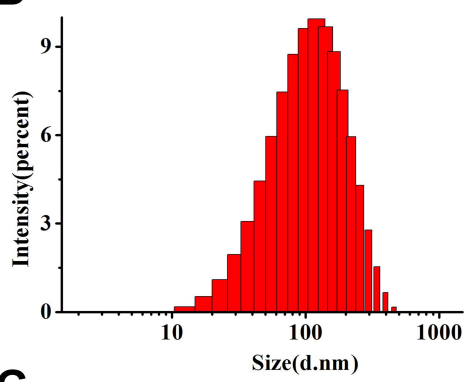

G

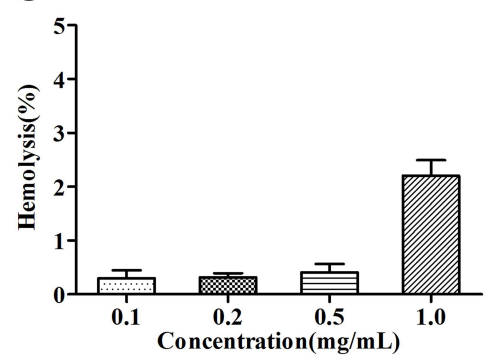

B

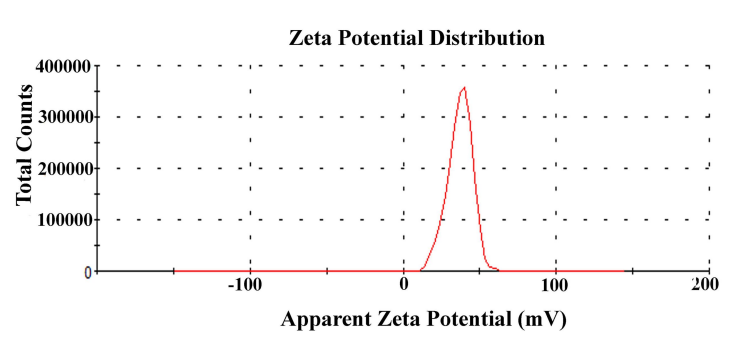

E

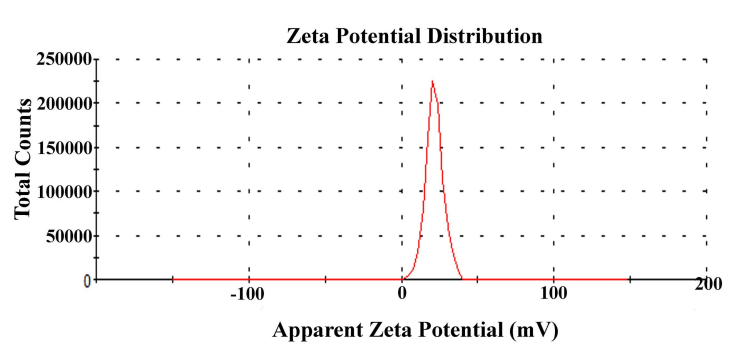

C

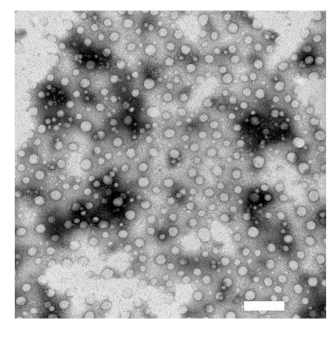

$\mathbf{F}$

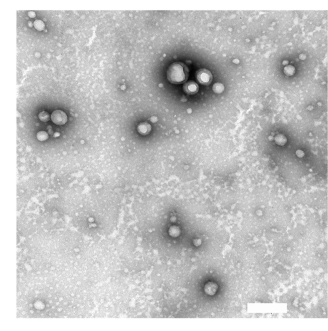

Figure 2 Size distribution by intensity (A), Zeta potential (B) and TEM images (C) of NPs-EPI; Size distribution by intensity (D), Zeta potential (E) and TEM images (F) of NPs-EPI/HAase; Hemolysis rate of carrier materials (G). Scale bars represent $200 \mathrm{~nm}$.

investigate some main obstacles that hinder the effective delivery of drugs to solid tumors. ${ }^{36}$ In the current study, tumor spheroids were treated with NPs-EPI combined with various concentrations $(0.05,0.1,0.5,1.0 \mathrm{mg} / \mathrm{mL}$ HAase, respectively) of HAase for 1 hour, 4 hours, and 8 hours, respectively, and the permeation of NPs-EPI was analyzed by confocal microscopy. As shown in Figure 4A-C, NPs-EPI /HAase possessed better penetration efficiency than NPsEPI, which was consistent with our previous report. ${ }^{24}$ The images obtained by confocal microscopy showed that the penetration depth of NPs-EPI was positively correlated with incubation time and the enzyme concentration. To define the penetration efficiency, we calculated the penetration percentage as the ratio of fluorescent intensity in the core and at the periphery of spheroid (Figure 4D-F). ${ }^{37}$ As shown in Figure 4E and F, when the incubation time was $4 \mathrm{~h}$ or $8 \mathrm{~h}$, the penetration percentage significantly increased with HAase concentration up to $0.5 \mathrm{mg} / \mathrm{mL}$. As shown in Figure 4F, when the incubation time was $8 \mathrm{~h}$, HAase concentration of $0.5 \mathrm{mg} / \mathrm{mL}$ showed the best penetration efficiency at $100 \mu \mathrm{m}$ sections. Taken into account these results, it was determined that $0.5 \mathrm{mg} / \mathrm{mL}$ HAase was the optimum concentration and was used in subsequent HA staining studies.

\section{Tumor Spheroids Structure Analyses}

The morphology of tumor spheroids as a function of HAase concentration was studied by fluorescence inverted microscopy (Figure 5A) and confocal microscopy (Figure 5B). Treating tumor spheroids with low concentration of HAase led to slight loosening of cells at the spheroid periphery, whereas treatment with high concentration of HAase (e.g. $0.5 \mathrm{mg} / \mathrm{mL}$ or $1 \mathrm{mg} / \mathrm{mL}$ ) dramatically affected the structural integrity of tumor spheroids and presented the sign of disintegration. This structural change may attribute to the degradation of HA in the ECM; thus, the ECM of tumor spheroids was no longer dense, and the structure of tumor spheroids became loose. 
A

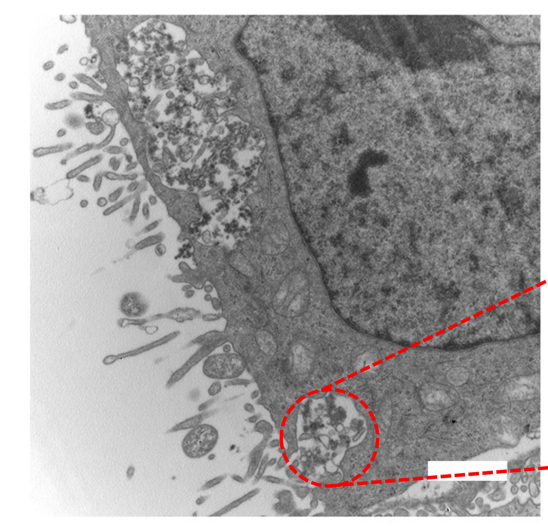

D

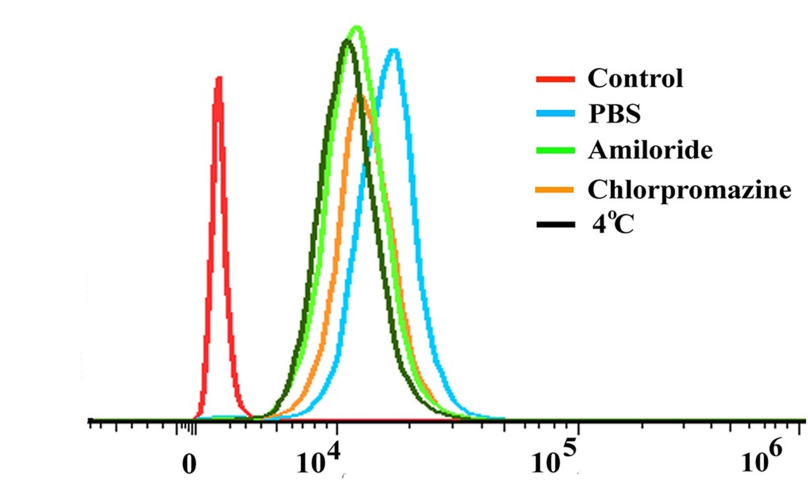

\section{D}

C

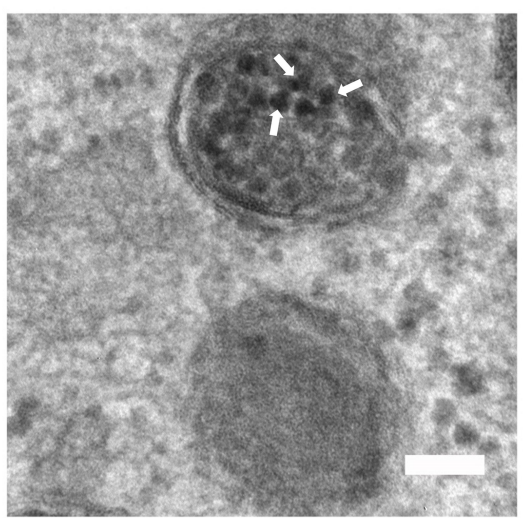

E

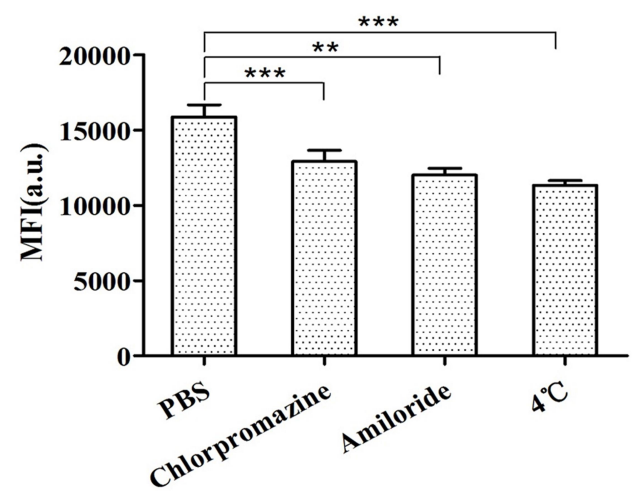

Figure 3 Representative TEM images of NPs-EPI in HepG2 spheroid sections. Arrows showed the drug-loaded micelles NPs-EPI. The micelles were discovered in the vesicles $(\mathbf{A}, \mathbf{B})$ and lysosomes $(\mathbf{C})$. Flow cytometric profiles of cellular uptake of NPs-EPI (D). The average fluorescence intensity of EPI in different treatment groups (E). $* * \mathrm{P}<0.01$, $* * * \mathrm{P}<0.001$. Data represent the mean $\pm S D(\mathrm{n}=3)$. Scale bars for $\mathrm{A}, \mathrm{B}$, and $\mathrm{C}$ represent $500 \mathrm{~nm}, 200 \mathrm{~nm}$, and I00 nm, respectively.

\section{HA Staining}

We hypothesize that HAase could degrade HA to facilitate small sized NPs-EPI penetrate into the tumor spheroid. To confirm the presence of HA in HepG2 tumor spheroids, spheroid sections were stained with HA binding protein (HABP) and Alexa Fluor 488-conjugated streptavidin and subsequently imaged by confocal microscopy. As shown in Figure 6A, HA labelling revealed that the ECM of HepG2 tumor spheroids contain high HA content, which may hinder the penetration of nanoparticles into the tumor spheroids. In comparison, treatment of spheroids with NPs/HAase (HAase $0.5 \mathrm{mg} / \mathrm{mL}$ ) led to a significant decrease in HA content as shown in Figure 6B. By degrading the HA in ECM, HAase could improve the penetration of NPs-EPI into tumor spheroids thus enhancing the uptake by cancer cells.

\section{Tumor Volume and Mice Body Weight Monitoring}

The increase of the penetration ability of nanoparticles in tumor tissues leads to more accumulation in tumor tissues and the stronger the inhibitory effect on tumor growth. Therefore, the changes of tumor volume and mice body weight were monitored during the construction of the tumor penetration model, which could reflect the strength of nanoparticles penetration ability from the side.

As shown in Figure 7A and C, all EPI formulations reduced the tumor volumes. Among them, the EPI group showed the strongest inhibition of tumor growth, which may due to the fact that EPI is a small molecule drug and it is easier to cross the extracellular matrix barrier of the tumor to kill cells than NPs. Compared with NPs-EPI group, NPs-EPI/HAase group showed better efficacy in tumor inhibition probably due to the high tumor accumulation/penetration and efficient cellular uptake. It should be noted that, although EPI group showed the strongest tumor inhibition, the body weight of the mice declined obviously during treatment indicating systemic toxicity to the mice (Figure 7B). In comparison, the group treated with NPsEPI/HAase showed no apparent weight loss, indicating better tolerance of mice to the treatment. 
A

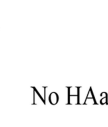

$0.05 \mathrm{mg} / \mathrm{mLHAase}$

$0.1 \mathrm{mg} / \mathrm{mL}$ HAase

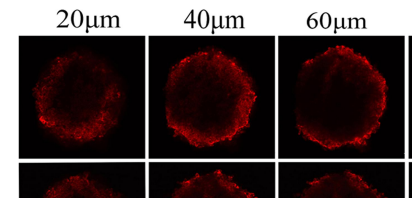

$0.5 \mathrm{mg} / \mathrm{mLHAase}$

$1 \mathrm{mg} / \mathrm{mLHAase}$

\section{B \\ NO HAase}

$0.05 \mathrm{mg} / \mathrm{mL}$ HAase

$0.1 \mathrm{mg} / \mathrm{mL}$ HAase

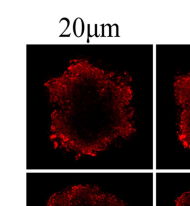

$40 \mu \mathrm{m}$

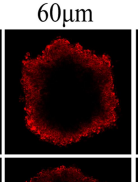

\section{.}
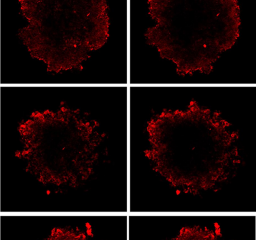

$0.5 \mathrm{mg} / \mathrm{mLHAase}$

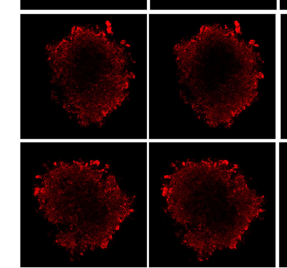

C

No HAase

$$
\text { t. }
$$

$0.05 \mathrm{mg} / \mathrm{mL}$ HAase

$0.1 \mathrm{mg} / \mathrm{mL}$ HAase

$0.5 \mathrm{mg} / \mathrm{mL}$ HAase

$1.0 \mathrm{mg} / \mathrm{mL}$ HAase
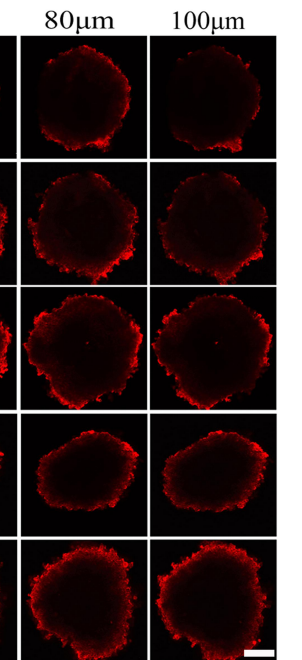

$80 \mu \mathrm{m} \quad 100 \mu \mathrm{m}$
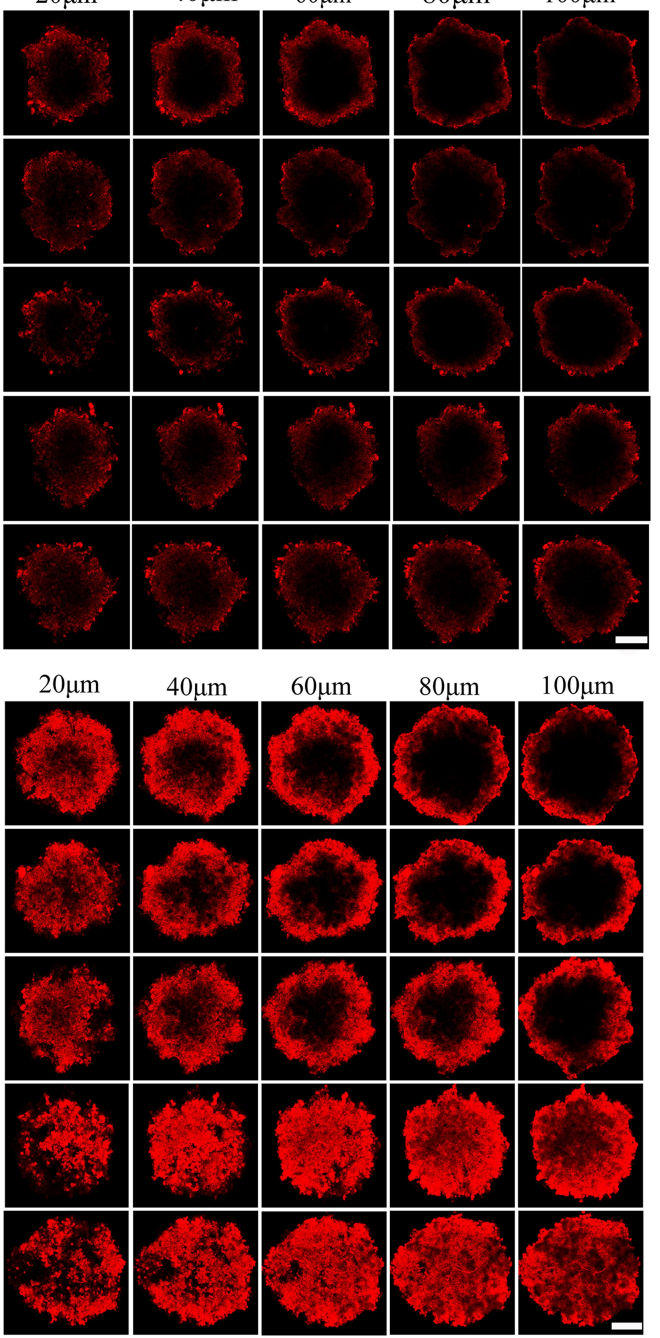

D

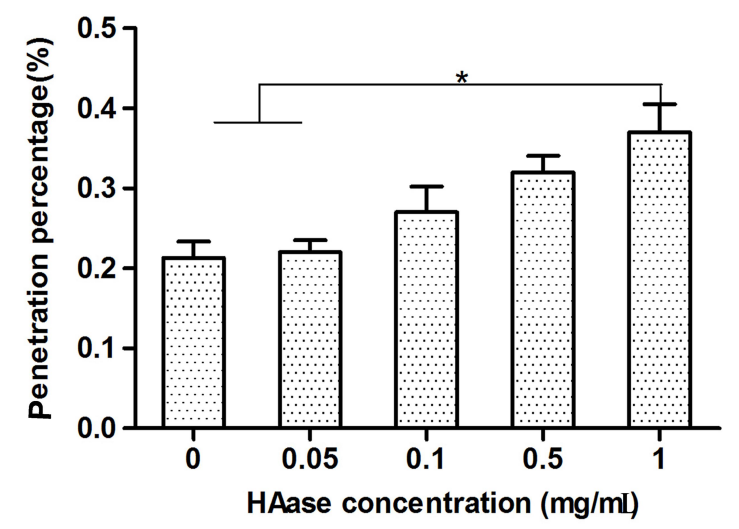

E

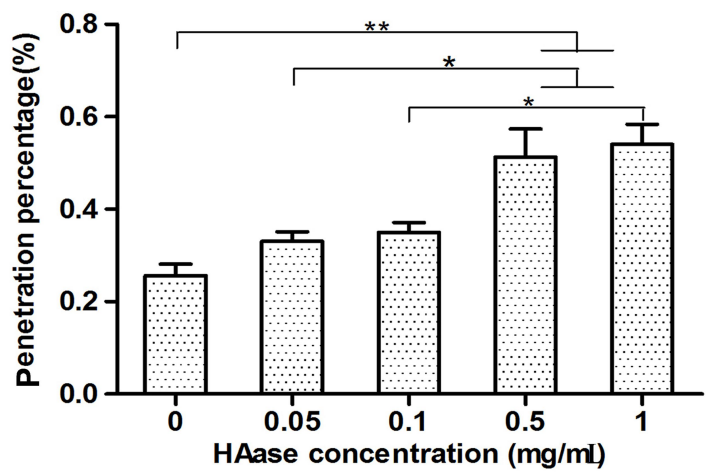

F

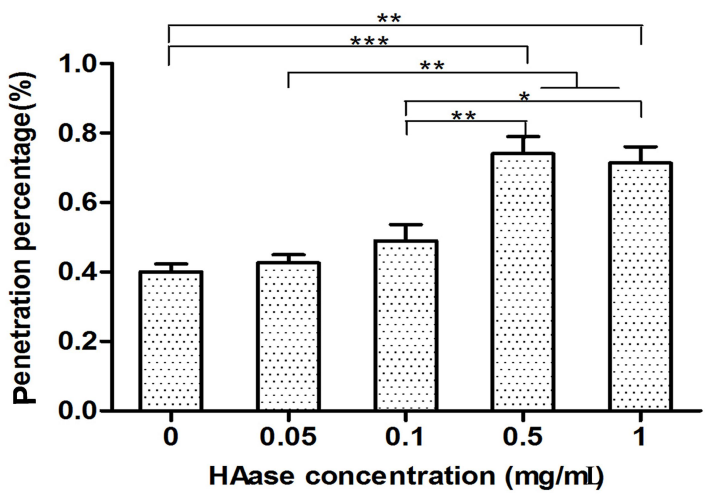

Figure 4 Confocal microscopy images of HepG2 tumor spheroids co-incubated with NPs-EPI, NPs-EPI/HAase (combined with $0.05,0.1,0.5$, I.0 mg/mL HAase, respectively) for I hour (A), 4 hours (B), and 8 hours (C). The penetration percentage of NPs-EPI and NPs-EPI/HAase (combined with 0.05, 0.I, 0.5, I.0 mg/mL HAase, respectively) for I hour (D), 4 hours (E), and 8 hours $(\mathbf{F})$ at $100 \mu \mathrm{m}$ sections. Data represent the mean \pm SD $(\mathrm{n}=3)$. $* \mathrm{P}<0.05$, $* * \mathrm{P}<0.0 \mathrm{I}$, $* * * \mathrm{P}<0.00 \mathrm{I}$. Scale bar represents $\mathrm{I00} \mu \mathrm{m}$. 


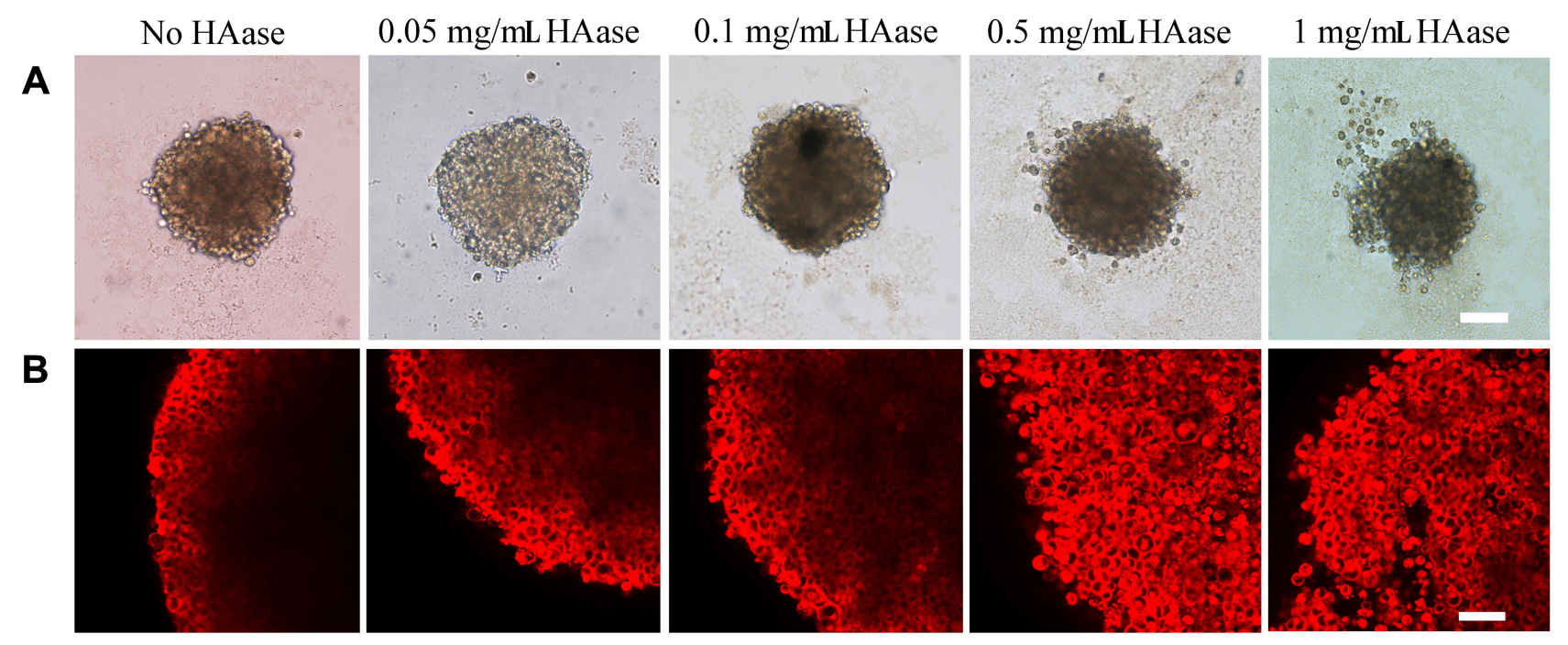

Figure 5 Fluorescence inverted microscopy images (A) and confocal microscopy images (B) of HepG2 tumor spheroids upon incubation with NPs-EPI, NPs-EPI/HAase (combined with $0.05,0.1,0.5,1.0 \mathrm{mg} / \mathrm{mL}$ HAase, respectively). Scale bars for A, B represent $200 \mu \mathrm{m}$ and $50 \mu \mathrm{m}$, respectively.

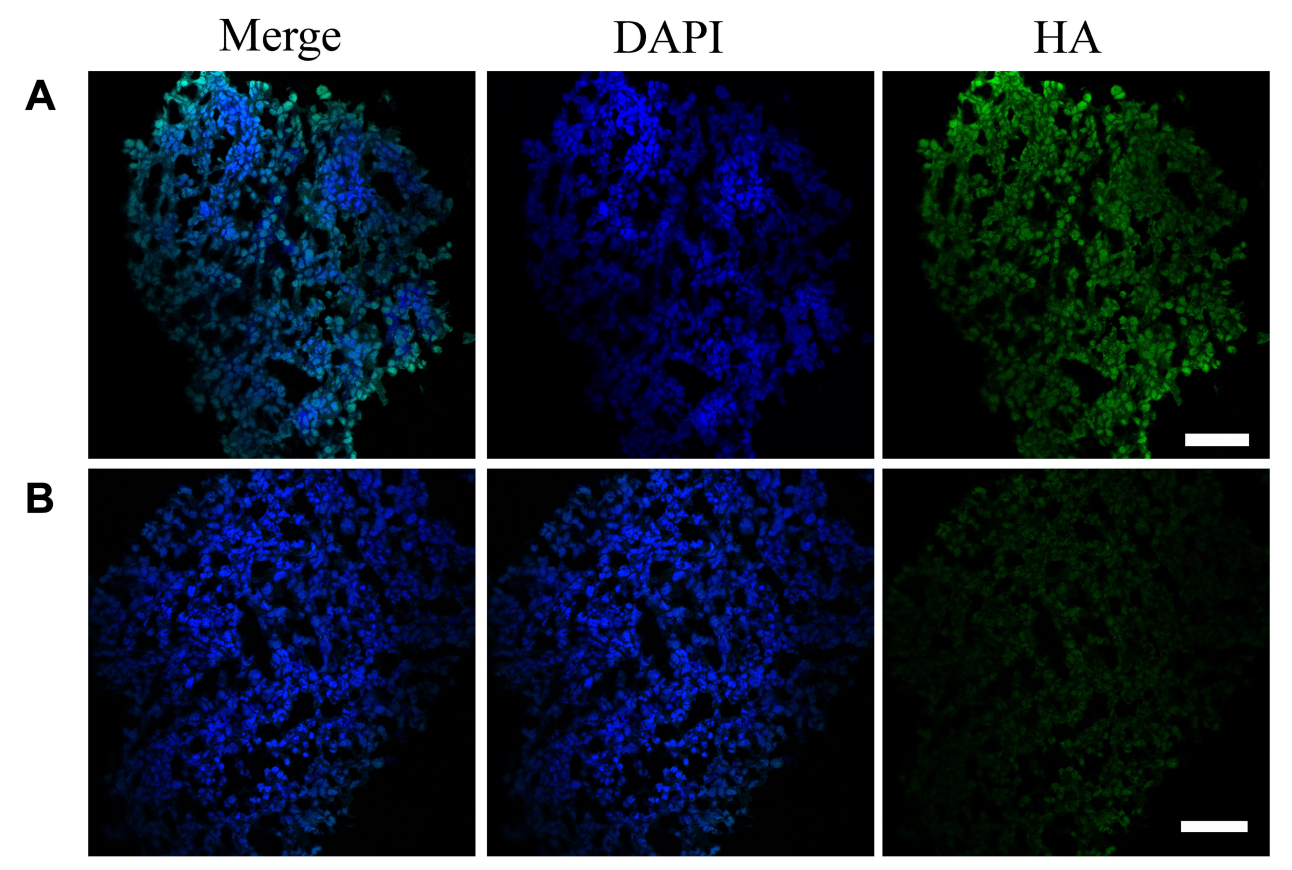

Figure 6 HA-stained cryosections of spheroids in the HAase-untreated group (A) and HAase-treated group (B). HAase-treated spheroids were exposed to NPs/HAase combined with $0.5 \mathrm{mg} / \mathrm{mL}$ of HAase for 8 hours before sectioning and staining. HA stain was showed in green and the nuclear stain, DAPI, was shown in blue. Scale bar represents $100 \mu \mathrm{m}$.

The EPI group was actually a positive control group, and we designed EPI to be administered in the form of nanoparticles in order to enhance its tumor-targeting ability when administered systemically. Our strategy avoids the side effects of systemic administration of EPI while enhancing the permeability of nanoparticles in tumor tissues. In future experiments, we will use more animal samples to study the antitumor ability of NPs-EPI/HAase in vivo.

\section{In vivo Tumor Penetration}

We demonstrated that the combination with HAase could improve penetration efficacy of NPs by transporting across the ECM, which was consistent with our previous report. ${ }^{24}$ In the current study, we further examined the content of HA and NPs in the tumor 4 hours or 12 hours after peritumoral injection. The HA were stained with HABP (green), the cell nuclei stained with DAPI (blue) and the 
A

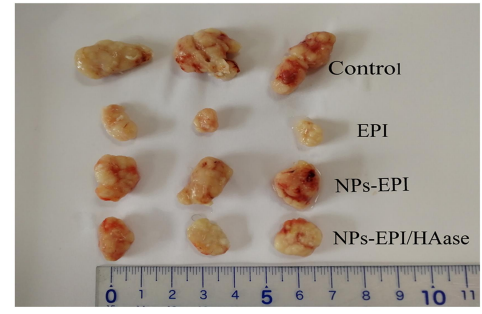

B

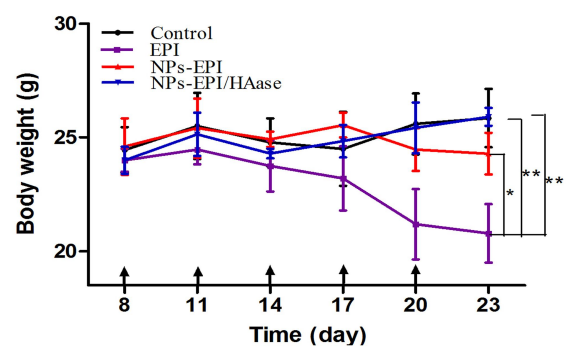

C

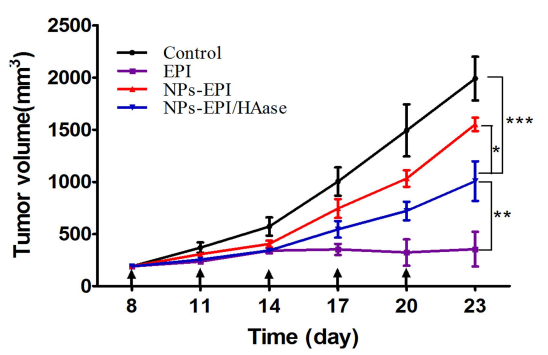

Figure 7 The changes of tumor volume and mice body weight during the construction of the tumor penetration model. (A) Photographs of tumors harvested from different groups on day 23. (B) Body weight changes in mice following different treatments within 23 days. (C) Tumor volume changes in time of mice treated with saline, EPI, NPs-EPI and NPs-EPI/HAase. The formulations were given on days $8,1 \mathrm{I}, 14,17$ and 20 at a dosage of EPI was $3 \mathrm{mg} / \mathrm{kg}$ while the concentration of HAase combined with micelles was $3 \mathrm{mg} / \mathrm{mL}$. Black arrows represent the time of injection. Data represent the mean $\pm S D(n=3) . * P<0.05, * * P<0.01, * * * P<0.001$.

NPs fluorescently labelled with DiD (red). As shown in Figure 8, for the control group, we observed abundant HA which was part of the main components of ECM in tumors and its accumulation could lead to increased IFP thus hindered drug diffusion. In the sample with 4 hours after injection of NPs-DiD, there were no obvious differences in terms of the HA green fluorescence intensity. In comparison, the green fluorescence of HA was obviously attenuated in the tumor after injection of NPs-DiD/HAase; meanwhile, the increase of $\mathrm{DiD}$ red fluorescence was also observed. This was due to HA degradation by HAase resulting in increased accumulation and penetration

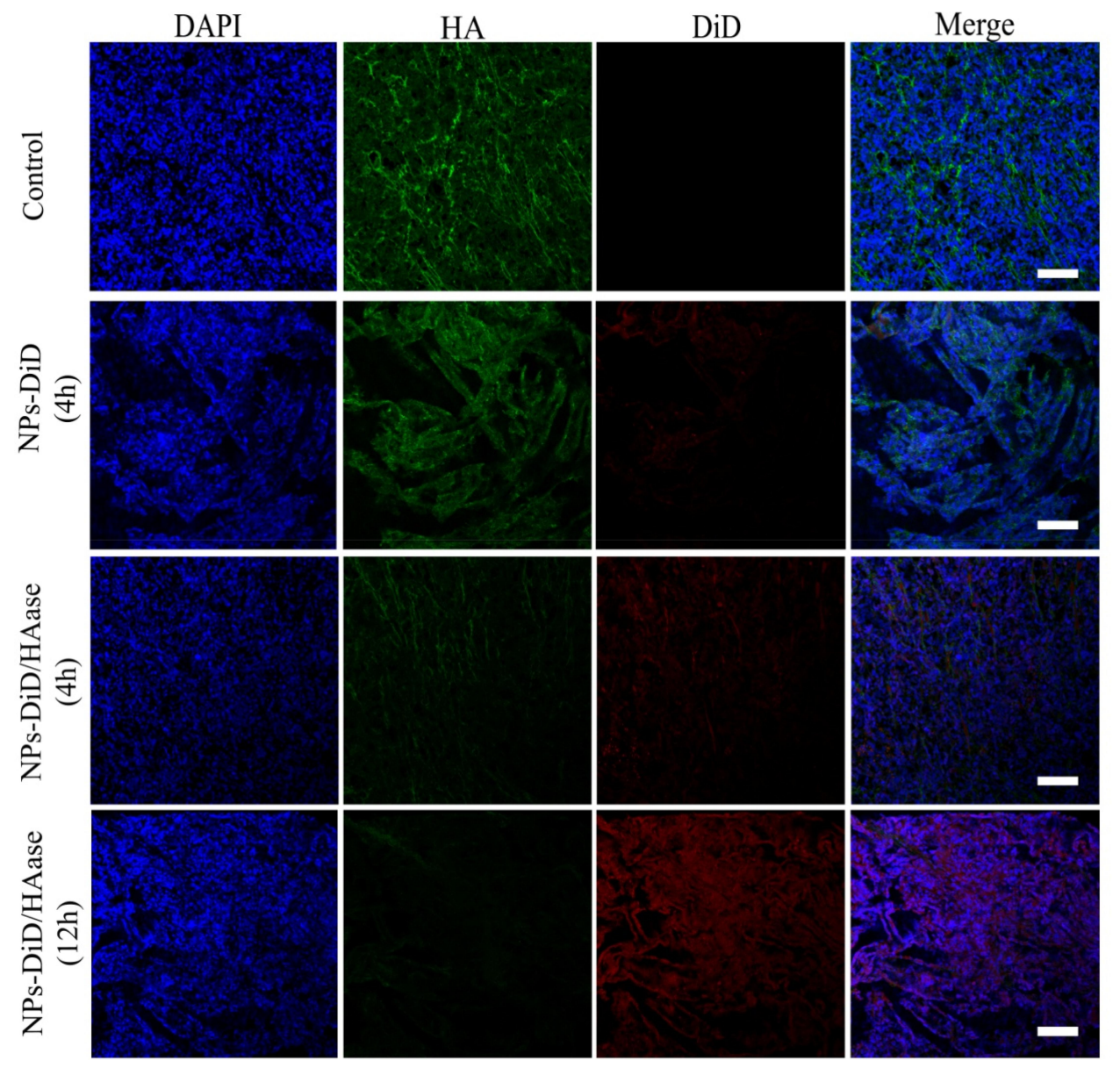

Figure 8 Tumor penetration behavior of NPs-DiD studied by confocal microscopy. The nuclei was stained with DAPI (blue), HA was stained with HABP (green), and NPsDiD was presented in red. Scale bar represents $100 \mu \mathrm{m}$. 
A

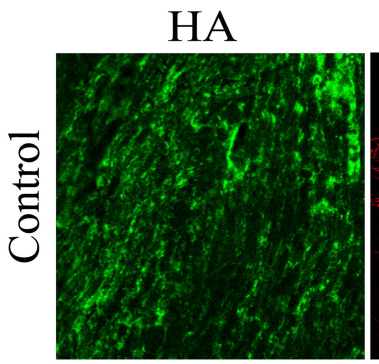

Collagen-1

$\alpha-\mathrm{SMA}$

CD31
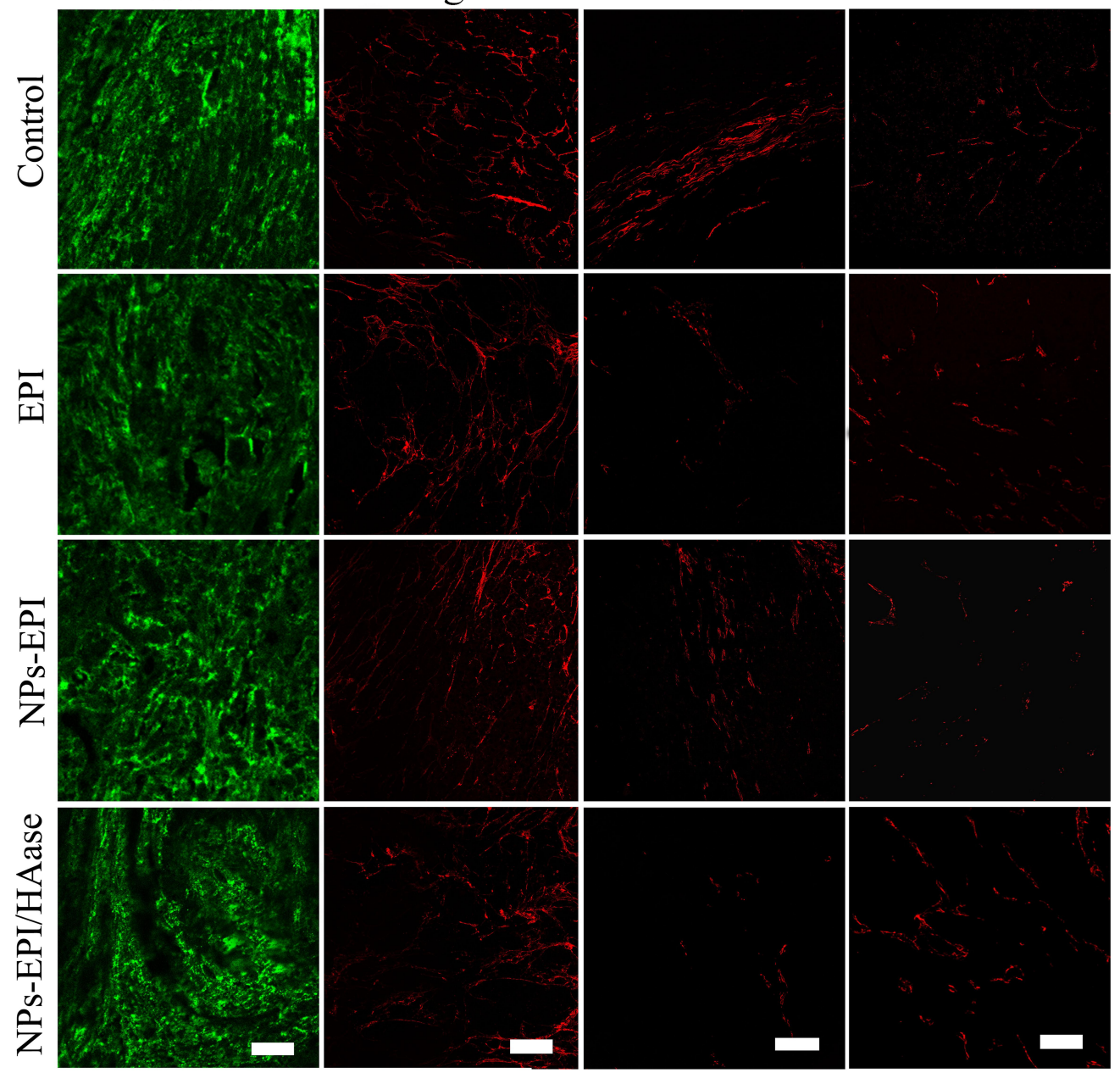

B
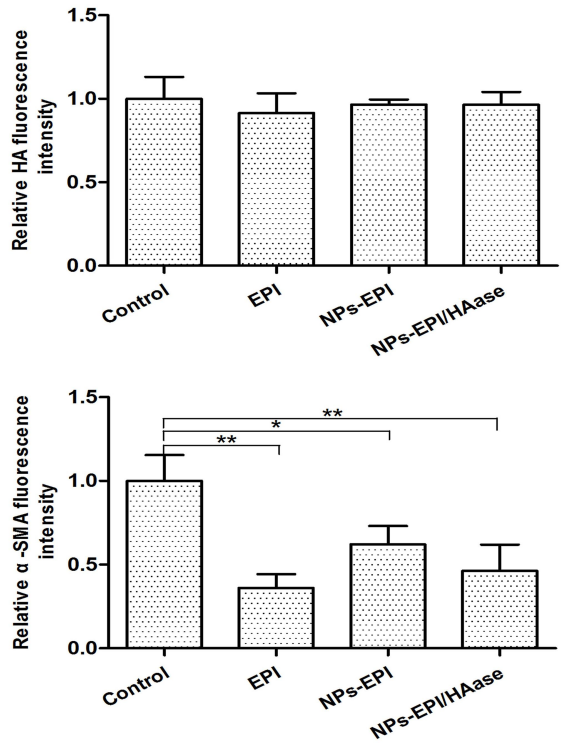

E
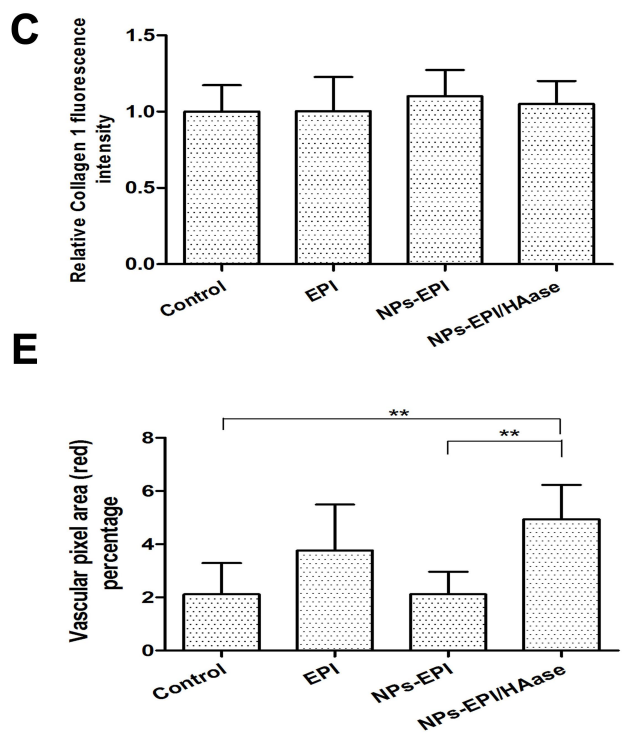

Figure 9 (A) Immunofluorescence imaging of tumor slices after treatment with saline, EPI, NPs-EPI and NPs-EPI/HAase. Saline-injected tumor slices were invoked as the control. The HA was stained with HABP, collagen I was stained with the anti-collagen I antibody, $\alpha$-SMA was stained with the anti- $\alpha$-SMA antibody, tumor blood vessels were stained with the anti-CD3I antibody, respectively. The nuclei of cells (blue) were stained with DAPI. Scale bar represents $100 \mu \mathrm{m}$. Quantitative analysis of HA (B), collagen I (C) and $\alpha$-SMA (D) fluorescence intensity used Image J. The average fluorescence intensity of tumors from saline-treated mice was set as I. (E) Statistic data of vascular pixel area (red) percentage of tumors slices used Image J. Values indicate mean $\pm S D$ ( $n=6$ from 3 tumor samples per study group). $* P<0.05$, $* * P<0.01$. 
of NPs in tumor tissues. As expected, it had been observed that the green fluorescence of HA became much weaker whereas the red fluorescence of DiD was more widely distributed in the tumor 12 hours after injection of NPsDiD/HAase.

\section{Penetration Mechanisms}

To investigate the penetration mechanism of NPs-EPI /HAase, we further examined the variation of tumor HA and collagen I, density and physiologic state of tumor vasculatures and activity of cancer-associated fibroblasts (CAFs) after the penetration models were constructed.

As shown in Figure 9A and B, HA staining did not show a significant difference in four groups after treatment. On one hand, within 15 days after the first administration, the tumor volume of nude mice in NPs-EPI /HAase group increased by 4-5 times, but the concentration of hyaluronidase we gave was still maintained at the initial low dose. On the other hand, it has been reported that hyaluronic acid is produced in large quantities during the rapid growth of tumors. ${ }^{30,38}$ These two factors resulted in no significant change in the amount of hyaluronic acid in several groups.

Collagen I is another main component of tumor ECM, and the decrease of collagen I content could also enhance the penetration of NPs in tumors and improve antitumor efficacy. ${ }^{39}$ In order to study whether the enhanced penetration of NPs-EPI/HAase in HepG2 tumors was due to the reduction of collagen I content, collagen I of sectioned tumor tissues was stained and examined. As shown in Figure 9A and $\mathrm{C}$, there was no significant difference in collagen I expression among the four groups, indicating that the enhanced penetration of NPs-EPI/HAase was not due to the reduction of collagen I by NPs.

In most solid tumors, CAFs not only interact with tumor cells to transmit signals of tumor progression but also synthesize and remodel tumor ECM including HA that hamper drug delivery. ${ }^{40}$ It has been reported that reduced CAFs activity by NP-carried drug could improve NP penetration in tumors due to the decreased ECM density. ${ }^{41}$ We chose protein marker $\alpha$-SMA to investigate the accumulation of CAFs in tumor, although CAFs consist of multiple resource subtypes, $\alpha$-SMA-positive fibroblasts could be regarded as the main CAFs. ${ }^{42}$ As shown in Figure 9A and D, compared with the control group, the red fluorescence intensity of $\alpha$-SMA in EPI, NPs-EPI, and NPs-EPI/HAase treatment group was significantly reduced, and no significant difference was found in the fluorescence intensity of $\alpha$-SMA between the three treatment groups, indicating that this decrease in CAFs activity may be mainly due to the toxicity of EPI. The result is consistent with the report that the survival of fibroblasts was reduced when the cells were exposed to epirubicin. ${ }^{43}$

The disruption of ECM in the tumors leads to a decrease in IFP, which directly affects the physiological state of the tumor vasculatures. ${ }^{44}$ The blood vasculatures of sectioned tumor tissues were stained with CD31. As shown in Figure 9A and E, by analyzing the density and physiologic state of tumor vasculatures, it was found that the density of blood vessels and the amount of dilated blood vessels were much higher after NPs-EPI/HAase treatment than that in the other groups, revealing that NPsEPI/HAase treatment could enhance the density of blood vessels and induce tumor vascular normalization due to the degradation of hyaluronic acid.

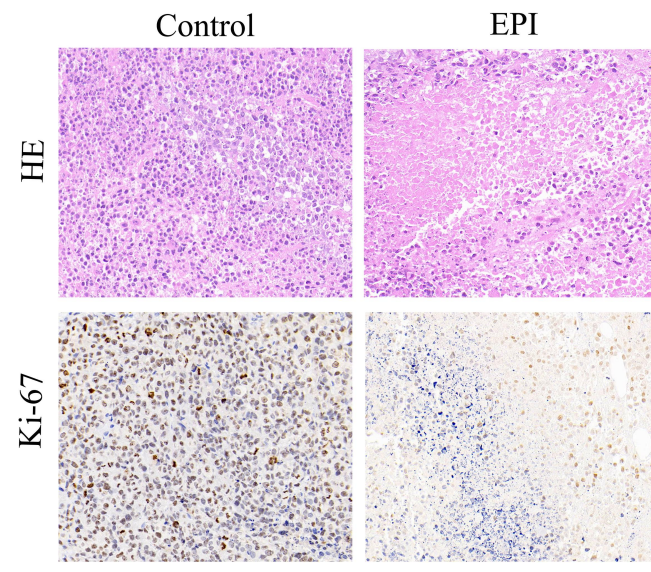

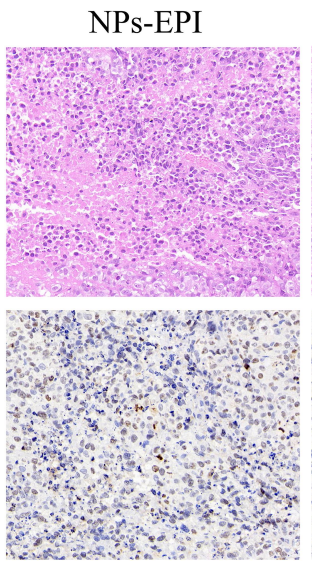

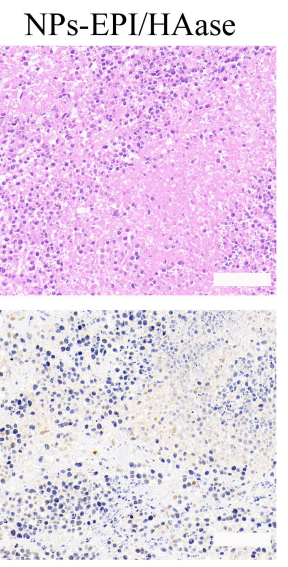

Figure $10 \mathrm{H} \& \mathrm{E}$ and $\mathrm{Ki}-67$ staining of the HepG2 tumor slices from mice treated with saline, EPI, NPs-EPI and NPs-EPI/HAase. Scale bar represents $100 \mu \mathrm{m}$. 


\section{Histological and Immunohistochemical Analysis}

After the tumor penetration model was constructed, tumors were harvested and $H \& E$ staining of tumor slices was performed to determine the tissue damage. As shown in Figure 10, both EPI and NPs-EPI/HAase treatments caused considerable tissue damage while saline group exhibited negligible tissue damage with intact nuclei morphology, this may due to EPI was easier to cross the extracellular matrix barrier of the tumor than NPs and the enhanced tumor penetration of NPs-EPI/HAase. At the same time, tumor slices from NPs-EPI group exhibited some degree of tissue damage compared with the control group. Ki-67 antigen staining is positive for brown granules in the nucleus and Ki-67 nuclear antigen expressed at certain stages of the cell cycle is involved in cell proliferation. ${ }^{45}$ As shown in Figure 10, the saline group had the highest number of Ki-67 positive cells. Compared with NPs-EPI group, NPs-EPI/HAase group exhibited lower level of tumor cell proliferation. All in all, due to the enhanced tumor penetration, NPs-EPI/HAase group exhibited the higher tissue damage and the lower amount of Ki-67 positive cells than NPs-EPI group.

\section{Conclusion}

In this study, we developed a hyaluronidase-embedded drug delivery system which could enhance tumor penetration by modulating the tumor microenvironment. We revealed that the hyaluronidase (HAase) can degrade HA to enhance the penetration efficacy of NPs. The NPs-EPI/HAase showed a better accumulation and deeper tumor penetration in HepG2 tumors, and leading to better inhibition of tumor growth. Overall, our studies suggested that coating nanoparticles with HAase may provide a simple but efficient strategy for nano-drug carriers to enhance solid tumor penetration and chemotherapeutic efficacy.

\section{Funding}

This work was supported by the National Natural Science Foundation of China [81673360, 81601591], Major Science and Technology Innovation Projects of Shandong Province [2018CXGC1408] and Science and Technology Projects for People's Livelihood of Qingdao [18-6-1-93nsh].

\section{Disclosure}

No conflict of interest was reported by the authors.

\section{References}

1. Niu Y, Zhu J, Li Y, et al. Size shrinkable drug delivery nanosystems and priming the tumor microenvironment for deep intratumoral penetration of nanoparticles. J Control Release. 2018;277:35-47. doi:10.1016/j.jconrel.2018.03.012

2. Cao J, Chen Z, Chi J, et al. Recent progress in synergistic chemotherapy and phototherapy by targeted drug delivery systems for cancer treatment. Artif Cells Nanomed Biotechnol. 2018;46(sup1):817-830. doi:10.1080/21691401.2018.1436553

3. Kratz F, Warnecke A. Finding the optimal balance: challenges of improving conventional cancer chemotherapy using suitable combinations with nano-sized drug delivery systems. J Control Release. 2012;164(2):221-235. doi:10.1016/j.jconrel.2012.05.045

4. Chen J, Ding J, Wang Y, et al. Sequentially responsive shell-stacked nanoparticles for deep penetration into solid tumors. Adv Mater. 2017;29(32):1701170. doi:10.1002/adma.201701170

5. Cui T, Yan Z, Qin H, et al. A sequential target-responsive nanocarrier with enhanced tumor penetration and neighboring effect in vivo. Small. 2019;15(42):e1903323. doi:10.1002/smll.201903323

6. Han M, Huang-Fu MY, Guo WW, et al. MMP-2-sensitive HA end-conjugated poly(amidoamine) dendrimers via click reaction to enhance drug penetration into solid tumor. ACS Appl Mater Interfaces. 2017;9(49):42459-42470. doi:10.1021/acsami.7b10098

7. Li HJ, Du JZ, Du XJ, et al. Stimuli-responsive clustered nanoparticles for improved tumor penetration and therapeutic efficacy. Proc Natl Acad Sci U S A. 2016;113(15):4164-4169. doi:10.1073/pnas. 1522080113

8. Ruan S, Cao X, Cun X, et al. Matrix metalloproteinase-sensitive size-shrinkable nanoparticles for deep tumor penetration and $\mathrm{pH}$ triggered doxorubicin release. Biomaterials. 2015;60:100-110. doi:10.1016/j.biomaterials.2015.05.006

9. Ostergaard L, Tietze A, Nielsen T, et al. The relationship between tumor blood flow, angiogenesis, tumor hypoxia, and aerobic glycolysis. Cancer Res. 2013;73(18):5618-5624. doi:10.1158/00085472.CAN-13-0964

10. Xiao W, Ruan S, Yu W, et al. Normalizing tumor vessels to increase the enzyme-induced retention and targeting of gold nanoparticle for breast cancer imaging and treatment. Mol Pharm. 2017;14 (10):3489-3498. doi:10.1021/acs.molpharmaceut.7b00475

11. Khawar IA, Kim JH, Kuh HJ. Improving drug delivery to solid tumors: priming the tumor microenvironment. J Control Release. 2015;201:78-89. doi:10.1016/j.jconrel.2014.12.018

12. Zhang YR, Lin R, Li HJ, et al. Strategies to improve tumor penetration of nanomedicines through nanoparticle design. Wiley Interdiscip Rev Nanomed Nanobiotechnol. 2019;11(1):e1519. doi:10.1002/ wnan.1519

13. Eikenes L, Tufto I, Schnell EA, et al. Effect of collagenase and hyaluronidase on free and anomalous diffusion in multicellular spheroids and xenografts. Anticancer Res. 2010;30(2):359-368.

14. Whatcott CJ, Han H, Posner RG, et al. Targeting the tumor microenvironment in cancer: why hyaluronidase deserves a second look. Cancer Discov. 2011;1(4):291-296. doi:10.1158/2159-8290.CD-11-0136

15. Sironen RK, Tammi M, Tammi R, et al. Hyaluronan in human malignancies. Exp Cell Res. 2011;317(4):383-391. doi:10.1016/j. yexcr.2010.11.017

16. Itano N, Zhuo L, Kimata K. Impact of the hyaluronan-rich tumor microenvironment on cancer initiation and progression. Cancer Sci. 2008;99(9):1720-1725. doi:10.1111/j.1349-7006.2008.00885.x

17. Camenisch TD, Spicer AP, Brehm-Gibson T, et al. Disruption of hyaluronan synthase-2 abrogates normal cardiac morphogenesis and hyaluronan-mediated transformation of epithelium to mesenchyme. J Clin Invest. 2000;106(3):349-360. doi:10.1172/JCI10272

18. Chanmee T, Ontong P, Itano N. Hyaluronan: a modulator of the tumor microenvironment. Cancer Lett. 2016;375(1):20-30. doi:10.1016/j.canlet.2016.02.031 
19. Toole BP, Hascall VC. Hyaluronan and tumor growth. Am J Pathol. 2002;161(3):745-747. doi:10.1016/S0002-9440(10)64232-0

20. Ropponen K, Tammi M, Parkkinen J, et al. Tumor cell-associated hyaluronan as an unfavorable prognostic factor in colorectal cancer. Cancer Res. 1998;58(2):342-347.

21. Knudson W. Tumor-associated hyaluronan. Providing an extracellular matrix that facilitates invasion. Am $J$ Pathol. 1996;148 (6):1721-1726.

22. Eikenes L, Tari M, Tufto I, et al. Hyaluronidase induces a transcapillary pressure gradient and improves the distribution and uptake of liposomal doxorubicin (Caelyx) in human osteosarcoma xenografts. $B r \quad J \quad$ Cancer. 2005;93(1):81-88. doi:10.1038/sj. bjc. 6602626

23. Platt VM, Szoka FC Jr. Anticancer therapeutics: targeting macromolecules and nanocarriers to hyaluronan or CD44, a hyaluronan receptor. Mol Pharm. 2008;5(4):474-486. doi:10.1021/mp800024g

24. Dai J, Han S, Ju F, et al. Preparation and evaluation of tumour microenvironment response multistage nanoparticles for epirubicin delivery and deep tumour penetration. Artif Cells Nanomed Biotechnol. 2018;46(sup2):860-873. doi:10.1080/21691401.2018. 1470528

25. Glasgow MD, Chougule MB. Recent developments in active tumor targeted multifunctional nanoparticles for combination chemotherapy in cancer treatment and imaging. J Biomed Nanotechnol. 2015;11 (11):1859-1898. doi:10.1166/jbn.2015.2145

26. Mao C, Xie X, Liu X, et al. The controlled drug release by pH-sensitive molecularly imprinted nanospheres for enhanced antibacterial activity. Mater Sci Eng C Mater Biol Appl. 2017;77:84-91. doi:10.1016/j.msec.2017.03.259

27. Jiang L, Liang Y, Huo Q, et al. Viral capsids mimicking based on $\mathrm{pH}$-sensitive biodegradable polymeric micelles for efficient anticancer drug delivery. J Biomed Nanotechnol. 2018;14(8):1409-1419. doi:10.1166/jbn.2018.2587

28. Huo Q, Liang $\mathrm{Y}, \mathrm{Lu} \mathrm{W}$, et al. Integrated metalloproteinase, $\mathrm{pH}$ and glutathione responsive prodrug-based nanomedicine for efficient target chemotherapy. J Biomed Nanotechnol. 2019;15(8):1673-1687. doi:10.1166/jbn.2019.2801

29. Zhu Y, Zhang J, Meng F, et al. cRGD/TAT dual-ligand reversibly cross-linked micelles loaded with docetaxel penetrate deeply into tumor tissue and show high antitumor efficacy in vivo. ACS Appl Mater Interfaces. 2017;9(41):35651-35663. doi:10.1021/acsami.7b12439

30. Zhou H, Fan Z, Deng J, et al. Hyaluronidase embedded in nanocarrier PEG shell for enhanced tumor penetration and highly efficient antitumor efficacy. Nano Lett. 2016;16(5):3268-3277. doi:10.1021/acs. nanolett.6b00820

31. Ge R, Cao J, Chi J, et al. $<\mathrm{p}>$ NIR-guided dendritic nanoplatform for improving antitumor efficacy by combining chemo-phototherapy. Int J Nanomedicine. 2019;14:4931-4947. doi:10.2147/IJN.S203171

32. Deng $\mathrm{C}$, Jia $\mathrm{M}$, Wei $\mathrm{G}$, et al. Inducing optimal antitumor immune response through coadministering iRGD with pirarubicin loaded nanostructured lipid carriers for breast cancer therapy. Mol Pharm. 2017;14(1):296-309. doi:10.1021/acs.molpharmaceut.6b00932
33. Balaji A, Jaganathan SK, Ismail AF, et al. Fabrication and hemocompatibility assessment of novel polyurethane-based bio-nanofibrous dressing loaded with honey and Carica papaya extract for the management of burn injuries. Int J Nanomedicine. 2016;11:4339-4355. doi:10.2147/IJN.S112265

34. Joo KI, Lei Y, Lee CL, et al. Site-specific labeling of enveloped viruses with quantum dots for single virus tracking. ACS Nano. 2008;2(8):1553-1562. doi:10.1021/nn8002136

35. Sahay G, Alakhova DY, Kabanov AV. Endocytosis of nanomedicines. J Control Release. 2010;145(3):182-195. doi:10.1016/j.jconrel. 2010.01 .036

36. Goodman TT, Ng CP, Pun SH. 3-D tissue culture systems for the evaluation and optimization of nanoparticle-based drug carriers. Bioconjug Chem. 2008;19(10):1951-1959. doi:10.1021/bc800233a

37. Hu G, Zhang H, Zhang L, et al. Integrin-mediated active tumor targeting and tumor microenvironment response dendrimer-gelatin nanoparticles for drug delivery and tumor treatment. Int $J$ Pharm. 2015;496(2):1057-1068. doi:10.1016/j.ijpharm.2015.11.025

38. Brekken C, de Lange Davies C. Hyaluronidase reduces the interstitial fluid pressure in solid tumours in a non-linear concentration-dependent manner. Cancer Lett. 1998;131(1):65-70. doi:10.1016/S03043835(98)00202-X

39. Pan A, Wang Z, Chen B, et al. Localized co-delivery of collagenase and trastuzumab by thermosensitive hydrogels for enhanced antitumor efficacy in human breast xenograft. Drug Deliv. 2018;25 (1):1495-1503. doi:10.1080/10717544.2018.1474971

40. Pankova D, Chen Y, Terajima M, et al. Cancer-associated fibroblasts induce a collagen cross-link switch in tumor stroma. Mol Cancer Res. 2016;14(3):287-295. doi:10.1158/1541-7786.MCR-15-0307

41. Zhang J, Miao L, Guo S, et al. Synergistic anti-tumor effects of combined gemcitabine and cisplatin nanoparticles in a stroma-rich bladder carcinoma model. J Control Release. 2014;182:90-96. doi:10.1016/j.jconrel.2014.03.016

42. Pang N, Li J, Sun A, et al. Prior anti-CAFs break down the CAFs barrier and improve accumulation of docetaxel micelles in tumor. Int J Nanomedicine. 2018;13:5971-5990. doi:10.2147/IJN.S171224

43. Henriksson R, Grankvist K. Protective effect of iron chelators on epirubicin-induced fibroblast toxicity. Cancer Lett. 1988;43 (3):179-183. doi:10.1016/0304-3835(88)90168-1

44. Gong H, Chao Y, Xiang J, et al. Hyaluronidase to enhance nanoparticle-based photodynamic tumor therapy. Nano Lett. 2016;16(4):2512-2521. doi:10.1021/acs.nanolett.6b00068

45. Kee N, Sivalingam S, Boonstra R, et al. The utility of Ki-67 and BrdU as proliferative markers of adult neurogenesis. J Neurosci Methods. 2002;115(1):97-105. doi:10.1016/S0165-0270(02)00007-9
International Journal of Nanomedicine

\section{Publish your work in this journal}

The International Journal of Nanomedicine is an international, peerreviewed journal focusing on the application of nanotechnology in diagnostics, therapeutics, and drug delivery systems throughout the biomedical field. This journal is indexed on PubMed Central, MedLine, CAS, SciSearch ${ }^{\mathbb{R}}$, Current Contents ${ }^{\mathbb{R}} /$ Clinical Medicine, $^{2}$
Journal Citation Reports/Science Edition, EMBase, Scopus and the Elsevier Bibliographic databases. The manuscript management system is completely online and includes a very quick and fair peer-review system, which is all easy to use. Visit http://www.dovepress.com/ testimonials.php to read real quotes from published authors. 